\title{
The Role of Menin in Hematopoiesis
}

\author{
Ivan Maillard and Jay L. Hess ${ }^{*}$
}

\begin{abstract}
In the hematopoietic system, menin was found to interact with MLL, a large protein encoded by the mixed linage leukemia gene that acts as a histone $\mathrm{H} 3$ merhyltransferase. The $M L L$ gene is a recurrent target for translocations in both acute myeloid and acute lymphoid leukemias. MLL gene rearrangements involve a variety of translocation partners, giving rise to MLL fusion proteins whose transforming ability is mediated through upregulated expression of Homeobox (Hox) genes as well as other targets. Recent work indicates that menin is an essential partner of MLL fusion proteins in leukemic cells and that it regulates normal hematopoiesis. In the absence of menin, steady-state hematopoiesis is largely preserved; however, menin-deficient hematopoietic stem cells are markedly deficient in situations of hematopoietic stress, such as during recovery after bone marrow transplantation. In leukemias driven by MLL fusion proteins, menin is essential for transformation and growth of the malignant cells. Thus, menin-MLL interactions represent a promising therapeutic target in leukemias with MLL rearrangements.
\end{abstract}

\section{Introduction}

\section{Menin Is Associated with MLL, a Histone Methyltransferase Rearranged in Leukemia}

Recognition of the role of menin in normal and neoplastic hematopoiesis arose from studies of the mixed lineage leukemia gene $M L L$, the mammalian homolog of Drosophila trithorax. MLL rearrangements are a common cause of both acute lymphoid and myeloid leukemias (Fig. 1). Among lymphoid leukemias the most common MLL rearrangements are the $t(4 ; 11)$ and $t$ $(11 ; 19)$ translocations, which are associated with pro-B-cell leukemias that express $M L L$ $A F 4$ and $M L L-E N L$ respectively. The most common MLL rearrangements in acute myeloid leukemias include the $\mathrm{t}(9 ; 11), \mathrm{t}(11 ; 19)$ and $\mathrm{t}(10 ; 11)$, which express $M L L-A F 9, M L L-E L L$ and $M L L-A F 10$ respectively. MLL rearrangements are also common in secondary acute lymphoid and myeloid leukemias arising following therapy with topoisomerase inhibitors such as etoposide. ${ }^{1}$ In all, more than 50 different translocations have been identified.

MLL (3968 aa) is proteolytically cleaved into two fragments before entering the nucleus. ${ }^{2,3}$ The amino terminus of $\left(\mathrm{MLL}^{\mathrm{N}}\right)$ is a $300 \mathrm{kD}$ protein that directly targets MLL to specific chromosomal sites including promoters and coding regions of Hox genes. These sequences span a short evolutionarily conserved N-terminal domain (NTD), three AT hooks, which bind the minor groove of DNA and a nonenzymatic DNA methyltransferase homology (DNMT) region that is pivotal for MLL binding to unmethylated CpG-rich DNA. ${ }^{4,5}$ The $\mathrm{C}$ terminus of $\mathrm{MLL}^{\mathrm{N}}$ contains several regions with high homology to trx. These include three cysteine-rich zinc finger domains (termed PHD for plant homeodomain) which flank an imperfect bromodomain, a domain implicated in binding to acetylated histones. ${ }^{2,3,6-8}$

C2009 Landes Bioscienceand Springer Science+Business Media

*Corresponding Author: Jay L. Hess—Department of Pathology, University of Michigan Medical School, 5249 Medical Sciences 1, 1301 Catherine Avenue, Ann Arbor, Michigan 48105 USA. jayhess@umich.edu. 
The $180 \mathrm{kD} \mathrm{MLL}^{\mathrm{C}}$ peptide noncovalently associates with $\mathrm{MLL}^{\mathrm{N}}$ and has potent transcriptional activating activity. ${ }^{2,3}$ Transcriptional activation by MLL involves a concerted series of histone modifications mediated by MLL ${ }^{\mathrm{C}}$. The MLL SET domain has intrinsic histone

methyltransferase activity specific for histone $\mathrm{H} 3$ lysine 4 and that this plays a major role in transcriptional activation. ${ }^{9,10}$ In addition, histone acetylation also contributes to transcriptional activation. The histone acetyltransferase (HAT) CBP is recruited by MLL via hydrophobic interactions in MLL aa 2829-2883. ${ }^{11,12}$ MLL also interacts with the histone acetyltransferase MOF, an interaction that is important for transcriptional activation. ${ }^{13}$

Studies by several laboratories have shown that MLL is associated with mammalian homologs of proteins in the yeast Set1 merhyltransferase complex. These include a core complex composed of hASH2, Rbb5, WDR5 and Dpy30. ${ }^{13-15}$ This complex associates with the MLL SET domain and, via WDR5, targets MLL to sites of histone H3 lysine 4 dimethylation. ${ }^{16}$ Importantly, the MLL complex also contains menin. ${ }^{17}$ The exact function of menin is yet to be determined, although it is clear that menin is directly involved with transcriptional regulation. Menin has been reported to repress transcriptional activation by transcription factors JunD ${ }^{18}$ and NF- $\mathrm{BB} .{ }^{19}$ However, our studies show that menin is involved in transcriptional activation. Menin interacts with the serine 5 phosphorylated form of RNA polymerase II (RNA pol II) and is required for transcription of target genes including the clustered Hox genes. ${ }^{14,20}$ Menin interacts with MLL via a domain that is conserved in all leukemogenic MLL fusion proteins and apparently recruits MLL to target loci.

The best understood targets of MLL are the clustered homeobox or Hox genes, which are transcription factors that specify segment identity and cell fate during development. Previous studies showed that $M l l(M l l=$ murine $M L L)$ positively regulates Hox gene expression during development ${ }^{21}$ because heterozygous $M l l$ knockout mice showed posterior shifts in Hox gene expression. Moreover, Mll knockout mice are embryonic lethals in which patterns of Hox expression initiate normally, but are not maintained past embryonic day 9.5, when Hox expression drops to undetectable levels, indicating a pivotal role for MLL in maintenance of Hox gene expression.

Work done by our laboratory and others has also identified additional MLL/menin targets that are outside of the clustered Hox genes. Building on insights gained from growth data and microarray gene expression analysis on Mll and menin knockout fibroblasts, we determined that MLL directly regulated the expression of the cyclin-dependent kinase inhibitors $p 27^{\text {Kip } 1}$ and $p 18^{I n k 4 c} .22 \mathrm{We}$ found that menin activates transcription via a mechanism involving recruitment of MLL to the $p 27^{K i p 1}$ and $p 18^{I n k 4 c}$ promoters and coding regions. Loss of function of MLL or menin, either through ablation or expression of mutant alleles found in patients, results in down regulation of $p 27^{K i p 1}$ and $p 18^{I n k 4 c}$ expression and deregulated cell growth. These findings were further extended by analyzing a series of pancreatic and parathyroid tumors from MEN1 patients. These studies confirmed a marked decrease in $p 27^{\text {Kip } 1}$ in tumoral tissues. In aggregate, our data suggest that regulation of CDK inhibitor transcription by cooperative interaction between menin and MLL plays a central role in menin's activity as a tumor suppressor. These findings have been subsequently confirmed by other investigators. $23-25$

\section{Role of Menin in Hematopoiesis}

Normal hematopoiesis is markedly impaired in the absence of Mll and significant, but not overlapping, hematopoietic defects have now been identified in conditional menin knockout mice.

In the embryo, Mll is required to establish normal primitive and definitive hematopoiesis. ${ }^{26-}$

${ }^{28}$ In addition, recent evidence shows that Mll is essential to support the homeostasis of adult 
hematopoietic progenitors. ${ }^{29,30}$ In one of the two reports investigating this question, loss of Mll led to rapid and profound hematopoietic failure. ${ }^{29}$ This was associated with an initial decrease in the quiescence of hematopoietic stem cells (HSCs), followed by HSC loss, as well as with downregulated expression of Hoxa9, Hoxa 7 and other clustered Hox genes. In the other report using an alternative approach to inactivate the $\mathrm{Mll}$ gene, steady-state hematopoiesis was less severely impaired, but Mll-deficient HSCs were markedly defective upon competitive transplantation into lethally irradiated hosts. ${ }^{30}$ The basis for this difference in phenotypic severity is unclear, although it was likely related to differences in genetic strategies and may have resulted from incomplete elimination of Mll function in the mice with the less severe phenotype. ${ }^{30}$ Altogether, this work identified key roles for Mll both in the establishment of hematopoiesis during embryonic development and its subsequent maintenance throughout life.

In view of these findings and because menin interacts with the Mll complex in leukemic cells, it was important to delineate the physiological impact of menin on normal hematopoiesis. Menin-deficient mice die during mid-gestation with multiple developmental defects. ${ }^{31}$ Therefore, evaluating the role of menin in hematopoiesis required the use of a conditional Menl allele. ${ }^{32} \mathrm{Men} 1$ inactivation in adult mice led to a modest decrease in the peripheral blood white cell count, as well as to a decreased ability of bone marrow progenitors to generate colonies in methylcellulose assays. ${ }^{33}$ Unlike in Mll-deficient mice, loss of menin did not lead to overt hematopoietic failure. We have now investigated in detail the function of menindeficient hematopoietic progenitors. ${ }^{34}$ In the absence of hematopoietic stress, menin-deficient mice were able to maintain normal numbers of primitive hematopoietic progenitors containing hematopoietic stem cells, nonself-renewing multipotent progenitors and myeloerythroid progenitors. However, although common lymphoid progenitors were preserved, numbers of downstream B lineage progenitors were significantly decreased in the bone marrow, indicating that the lymphoid lineage is particularly sensitive to the loss of menin. In contrast to the mild defects observed during steady-state hematopoiesis, menin-deficient hematopoietic stem cells had a severely impaired repopulation potential in competitive transplantation assays and were also defective after drug-mediated chemoablation. Altogether, this discrepancy between a relatively well preserved steady-state hematopoiesis and profoundly abnormal HSC function after transplantation or chemoablation points to a specific role of menin in the adaptive response of HSCs to hematopoietic stress, a situation that involves the recruitment of quiescent HSCs into a burst of rapid proliferation.

The preservation of relatively normal hematopoiesis at steady-state in the absence of menin contrasts with the profound hematopoietic failure reported after loss of Mll, suggesting that menin may be absolutely required only for a subset of Mll's functions. ${ }^{29,33,34}$ It remains to be determined if other proteins can substitute for menin to support Mll function in certain conditions, or if Mll can exert some of its effects totally independently of menin. An improved understanding of menin's precise role in the Mll complex during transcriptional regulation will be important to answer this question.

The relevant target genes of menin in the hematopoietic system remain to be identified. Hoxa 9 deficiency causes defects in HSC and lymphoid progenitor function that share characteristics with the defects observed after menin loss. ${ }^{35,36}$ Therefore, it was tempting to speculate that reduced Hoxa9 expression would account for at least some of the defects of menin-deficient progenitors. Intriguingly, we found that Hoxa 9 expression was normal in menin-deficient progenitor fractions containing HSCs. ${ }^{34}$ These findings indicate that Mll and other regulatory inputs can maintain Hoxa9 expression without menin in steady-state conditions. However, maintenance or induction of Hoxa9 expression during a proliferative burst associated with hematopoietic stress may require menin-dependent epigenetic changes. Alternatively, the physiological role of menin in HSCs may not require Hoxa9 at all and thus be dissociated from its role in supporting MLL fusion protein-mediated transformation. 
Although it is clear that Hox genes are regulated by Mll and mediate many of its effects during transformation, the downstream genes that mediate Mll's effects in normal hematopoietic cells have also not been formally identified. Loss-of-function approaches have shown that the individual Hox genes examined so far do not support hematopoietic functions that are as prominent as the overall effect of Mll. Future work will have to establish if a combination of Hox genes regulated by Mll mediates its hematopoietic functions, or if Mll acts predominantly through non-Hox target genes.

The specific involvement of menin in HSC function during hematopoietic stress has several practical consequences. First, study of menin-deficient progenitors may give important insights in the regulation of the complex HSC response to situations of hematopoietic stress. This adaptive response is still poorly understood, yet it is functionally critical in many situations that are relevant to human health, such as hematopoietic recovery after chemotherapy or bone marrow transplantation. Of particular interest is the regulation of epigenetic changes that must occur in HSCs in this context and that may underlie the ability of HSCs to maintain expression of a functional stem cell program even while undergoing several rounds of rapid self-renewal divisions.

\section{Role of Menin in Leukemogenesis}

All the MLL fusion proteins examined to date upregulate expression of Hoxa9 and Meis 1 and this appears to be pivotal for leukemogenesis. Hox genes including Hoxa7 and $a 9$ and the Hox cofactor Meis1 are normally only expressed in early Scal+Lin-hematopoietic stem cells and then their expression is rapidly downregulated. ${ }^{37-40}$ Although MLL is expressed throughout hematopoietic differentiation, normally Hox gene and Meis 1 expression is physiologically down modulated. In the presence of MLL fusion proteins, this mechanism is perturbed. In keeping with this, human leukemias with MLL rearrangements, either lymphoidor myeloid, consistently express HOXA7, HOXA9 and MEIS1. ${ }^{41-43}$ Experimental models provide strong evidence that upregulation of Hox genes, particularly Hoxa 9 and Meis 1 , accounts for MLL fusion protein leukemogenicity. Hoxa 7 and Hoxa 9 are consistently expressed in leukemias arising in $\mathrm{BXH} 2$ as a result of retroviral integration. ${ }^{44,45}$ Notably, more than $95 \%$ of leukemias with $H o x a 7$ and $a 9$ over expression showa second integration resulting in over expression of Meisl. Cotransduction of Hoxa 9 and Meisl immortalizes hematopoietic progenitors in vitro and rapidly accelerates leukemia development in transplanted mice. ${ }^{37}$ These results are further supported by the inability of MLL fusion proteins to transform Hoxa9 knockout bone marrow. ${ }^{46}$

Menin is required for transcriptional activation and transformation by MLL fusion proteins. The protein binds to the N terminal 44 aa of MLL that are remote from the SET domain or MOF interaction domains. Previously we showed that menin interacts with the serine 5 phosphorylated form of RNA polymerase $\mathrm{II}^{20}$ and in addition found that in fibroblasts menin appears to be important for recruitment of the MLL methyltransferase complex to target promoters. ${ }^{10}$ It is likely that similar mechanisms are operative in hematopoietic cells. Deletion of the menin interaction domain from MLL fusion proteins results in complete loss of immortalizing ability. Furthermore we showed that dominant negative inhibitors ofthe MLLmenin interaction, which were derived from N-terminal MLL peptides inhibited the growth of MLL transformed cells (Fig.2). This is accompanied by down regulation of MLL targets including Hox genes and Meis $1 .{ }^{47}$

\section{Conclusion}

Menin functions as an essential partner of MLL proteins within a large multiprotein complex with homology to the yeast Set1 methyltransferase complex. Although the precise biochemical 
mechanisms of menin's action remain to be fully investigated, it is clear that menin contributes to MLL-mediated Histone 3 Lysine 4 methylation at target gene loci. Menin regulates the homeostasis of normal hematopoietic progenitors. In addition, menin appears essential to mediate the transcriptional effects of MLL fusion proteins in leukemic cells, such as upregulation of Hox gene expression. As menin has only modest effects on hematopoietic stem cells in steady-state conditions, our findings suggest the existence of a therapeutic window to target the menin-MLL interaction in leukemia stem cells while sparing adjacent normal stem cells, at least in the absence of hematopoietic stress. These findings suggest that targeting the MLL-menin interaction is a promising target for leukemias with MLL rearrangements and possibly other leukemias with high level Hox and Meisl expression.

\section{References}

1. Felix CA. Secondary leukemias induced by topoisomerase-targeted drugs. Blochim Biophys Acta 1998;1400:233-55.

2. Yokoyama A, Kitabayashi I, Ayton PM, et al. Leukemia proto-oncoprotein MLL is proteolytically processed into 2 fragments with opposite transcriptional properties. Blood 2002;100:3710-8. [PubMed: 12393701]

3. Nakamura T, Mori T, Tada S, et al. ALL-1 is a histone methyltransferase that assemblesa supercomplex of proteins involved in transcriptional regulation. Mol Cell 2002;10:1119-28. [PubMed: 12453419]

4. Birke M, Schreiner S, Garcia-Cuellar MP, et al. The MT domain of the proto-oncoprotein MLL binds to CpG-containing DNA and discriminates against methylation. Nucleic Acids Res 2002;30:958-65. [PubMed: 11842107]

5. Ayton PM, Chen EH, Cleary ML. Binding to nonmethylated CpG DNA is essential for target recognition, transactivation and myeloid transformation by an MLL oncoprotein. Mol Cell Bio 2004;24:10470-8. [PubMed: 15542854]

6. Schultz DC, Friedman JR, Rauscher FJ 3rd. Targeting histone deacetylase complexes via KRAB-zinc finger proteins: the PHD and bromodomains of KAP-1 form a cooperative unit that recruits a novel isoform of the Mi-2alpha subunit of NuRD. Genes Dev 2001;15:428-43. [PubMed: 11230151]

7. Fair K, Anderson M, Bulanova E, et al. Protein interactions of the MLL PHD fingers modulate MLL target gene regulation in human cells. Mol Cell Bioi 2001;21:3589-97.

8. Filetici P, PO, Ballario P. The bromodomain: a chromatin browser? Front Biosci 2001;6:866-76.

9. Milne TA, Briggs SD, Brock HW, et al. MLL targets SET domain methyltransferase activity to hox gene promoters. Mol Cell 2002;10:1107-17. [PubMed: 12453418]

10. Milne TA, Dou Y, Martin ME, et al. MLL associates specifically with a subset of transcriptionally active target genes. Proc Nat Acad Sci USA 2005;102:14765-70. [PubMed: 16199523]

11. Ernst P, Wang J, Huang M, et al. MLL and CREB bind cooperatively to the nuclear coactivator CREBbinding protein. Mol Cell Biol 2001;21:2249-58. [PubMed: 11259575]

12. Petruk S, Sedkov Y, Smith S, et al. Trithorax and dCBP acting in a complex to maintain expression of a homeotic gene. Science 2001;294:1331-4. [PubMed: 11701926]

13. Dou Y, Milne TA, Tackett AJ, et al. Physical association and coordinate function of the H3 K4 methyltransferase MLL1 and the H4 K16 acetyltransferase MOF. Cell 2005;121:873-85. [PubMed: 15960975]

14. Yokoyama A, Wang Z, Wysocka J, et al. Leukemia proto-oncoprotein MLL forms a SET1-like histone merhyltransferase complex with menin to regulate hox gene expression. Mol Cell Biol 2004;24:5639-49. [PubMed: 15199122]

15. Milne TA, Hughes CM, Lloyd R, et al. Menin and MLL cooperatively regulate expression of cyclindependent kinase inhibitors. Proc Nat Acad Sci USA 2005;102:749-54. [PubMed: 15640349]

16. Wysocka J, Swigut T, Milne TA, et al. WDR5 associates with histone H3 methylated at K4 and is essential for H3 K4 methylation and vertebrate development. Cell 2005;121:859-72. [PubMed: 15960974]

17. Agarwal SK, Lee Burns A, Sukhodolets KE, et al. Molecular pathology of the MEN1 gene. Ann N Y Acad Sci 2004;1014:189-98. [PubMed: 15153434] 
18. Agarwal SK, Guru SC, Heppner C, et al. Menin interacts with the AP1 transcription factor JunD and represses JunD-activated transcription. Cell 1999;96:143-52. [PubMed: 9989505]

19. Heppner C, Bilimoria KY, Agarwal SK, et al. The tumor suppressor protein menin interacts with NFkappaB proteins and inhibits NF-kappaB-mediated transactivation. Oncogene 2001;20:4917-25. [PubMed: 11526476]

20. Hughes CM, Rozenblatt-Rosen O, Milne TA, et al. Menin associates with a trithorax family histone methyltransferase complex and with the hoxc8 locus. Mol Cell 2004;13:587-97. [PubMed: 14992727]

21. Yu BD, Hess JL, Horning SE, et al. Altered hox expression and segmental identity in Mll-mutant mice. Nature 1995;378:505-8. [PubMed: 7477409]

22. Milne TA, Hughes CM, Lloyd R, et al. Menin and MLL cooperatively regulate expression of cyclindependent kinase inhibitors. Proc Natl Acad Sci USA 2005;102:749-54. [PubMed: 15640349]

23. Karnik SK, Hughes CM, Gu X, et al. Menin regulates pancreatic islet growth by promoting histone methylation and expression of genes encoding p27Kip1 and p18INK4c. Proc Nat Acad Sci USA 2005;102:14659-64. [PubMed: 16195383]

24. Scacheri PC, Davis S, Odom DT, et al. Genome-wide Analysis of menin binding provides insights into MEN1 tumorigenesis. PLoS Genetics 2006;2:e51. [PubMed: 16604156]

25. Schnepp RW, Chen YX, Wang H, et al. Mutation of tumor suppressor gene men 1 acutely enhances proliferation of pancreatic islet cells. Cancer Res 2006;66(11):5707-15. [PubMed: 16740708]

26. Hess JL, Yu BD, Li B, et al. Defects in yolk sac hematopoiesis in Mll-null embryos. Blood 1997;90:1799-806. [PubMed: 9292512]

27. Yagi H, Deguchi K, Aono A, et al. Growth disturbance in fetal liver hematopoiesis of Mll-mutant mice. Blood 1998;92:108-17. [PubMed: 9639506]

28. Ernst P, Fisher JK, Avery W, et al. Definitive hematopoiesis requires the mixed-lineage leukemia gene. Dev Cell 2004;6:437-43. [PubMed: 15030765]

29. Jude CD, Climer L, Xu D, et al. Unique and independent roles for MLL in adult hematopoietic stem cells and progenitors. Cell Stem Cell 2007;1:324-337. [PubMed: 18371366]

30. McMahon KA, Hiew SYL, Hadjur S, et al. Mll has a critical role in fetal and adult hematopoietic stem cell self-renewal. Cell Stem Cell 2007;1:338-345. [PubMed: 18371367]

31. Bertolino P, Radovanovic I, Casse H, et al. Genetic ablation of the tumor suppressor menin causes lethality at mid-gestation with defects in multiple organs. Mech Dev 2003;120:549-60. [PubMed: 12782272]

32. Crabtree JS, Scacheri PC, Ward JM, et al. Of mice and MEN1: Insulinomas in a conditional mouse knockout. Mol Cell Biol 2003;23:6075-85. [PubMed: 12917331]

33. Chen Y-X, Yan J, Keeshan K, et al. The tumor suppressor menin regulates hematopoiesis and myeloid transformation by influencing hox gene expression. Proc Natl Acad Sci USA 2006;103:1018-23. [PubMed: 16415155]

34. Maillard I, Chen Y, Tubbs AT, et al. Menin regulates the function of hematopoietic stem cells and lymphoid progenitors. Blood 2007;110:379a.

35. Lawrence HJ, Helgason CD, Sauvageau G, et al. Mice bearing a targeted interruption of the homeobox gene HOXA9 have defects in myeloid, erythroid and lymphoid hematopoiesis. Blood 1997;89:192230. [PubMed: 9058712]

36. Lawrence HJ, Christensen J, Fong S, et al. Loss of expression of the hoxa-9 homeobox gene impairs the proliferation and repopulating ability of hematopoietic stem cells. Blood 2005;106:3988-94. [PubMed: 16091451]

37. Kroon E, Krosl J, Thorsteinsdottir U, et al. Hoxa9 transforms primary bone marrow cells through specific collaboration with meis1a but not Pbx1b. EMBO J 1998;17:3714-25. [PubMed: 9649441]

38. Magli MC, Largman C, Lawrence HJ. Effects of HOX homeobox genes in blood cell differentiation. J Cell Physiol 1997;173:168-77. [PubMed: 9365517]

39. Lawrence HJ, Sauvageau G, Humphries RK, et al. The role of HOX homeobox genes in normal and leukemic hematopoiesis. Stem Cells 1996;14:281-91. [PubMed: 8724694] 
40. Pineault N, Helgason CD, Lawrence HJ, et al. Differential expression of hox, meis1 and Pbx1 genes in primitive cells throughout murine hematopoietic ontogeny. Exp Hematol 2002;30:49-57. [PubMed: 11823037]

41. Armstrong SA, Staunton JE, Silverman LB, et al. MLL translocations specify a distinct gene expression profile that distinguishes a unique leukemia. Nat Genet 2002;30:41-7. [PubMed: 11731795]

42. Rozovskaia T, Feinstein E, Mor O, et al. Upregulation of Meis1 and HoxA9 in acute lymphocytic leukemias with the $t(4: 11)$ abnormality. Oncogene 2001;20:874-8. [PubMed: 11314021]

43. Yeoh EJ, Ross ME, Shurtleff SA, et al. Classification, subtype discovery and prediction of outcome in pediatric acute lymphoblastic leukemia by gene expression profiling. Cancer Cell 2002;1:133-43. [PubMed: 12086872]

44. Nakamura T, Largaespada DA, Shaughnessy JD Jr, et al. Cooperative activation of hoxa and Pbx1related genes in murine myeloid leukaemias. Nat Genet 1996;12:149-53. [PubMed: 8563752]

45. Moskow JJ, Bullrich F, Huebner K, et al. Meis1, a PBX1-related homeobox gene involved in myeloid leukemia in BXH-2 mice. Mol Cell Biol 1995;15:5434-43. [PubMed: 7565694]

46. Ayton PM, Cleary ML. Molecular mechanisms of leukemogenesis mediated by MLL fusion proteins. Oncogene 2001;20:5695-707. [PubMed: 11607819]

47. Caslini C, Yang Z, El-Osta M, et al. Interaction of MLL amino terminal sequences with menin is required for transformation. Cancer Res 2007;67:7275-83. [PubMed: 17671196] 


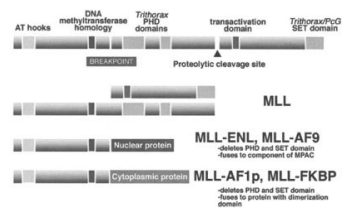

Figure 1.

Schematic of MLL and the two general types of MLL fusion proteins. The most common translocations fuse MLL to nuclear translocation partners with transcriptional activating activity. Many of these are components of the MLL Partner Activating Complex (MPAC) (see text and Fig. 2). Less commonly, MLL is fused to a translocation partner that transforms through dimerization of the truncated MLL molecule. MLL-FKBP is an experimental fusion protein that transforms only in the presence of synthetic dimerizer. MLL and both classes of MLL fusion proteins interact with menin via sequences in the extreme amino terminus. 


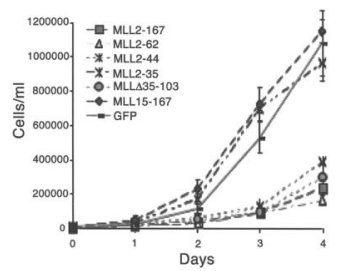

Figure 2.

Transduction of leukemic cells with expression vectors that coexpress green fluorescent protein and peptides that inhibit the MLL-menin interaction inhibits growth. Growth curve analysis of cells purified by sorting for GFP expression. All peptides that inhibit MLL-menin interaction (MLL2-167, MLL2-62, MLL 2-44, MLLA35-103 inhibit growth while those that do not block the interaction (MLL2-35, MLL15-167, GFP) do not. Points show means of triplicate experiments, while bars show standard deviation. (Reproduced with permission from Caslini et al. Cancer Res 67:7275-83, 2007). 